\title{
Relationship between electrodiagnostic severity and neuropathic pain assessed by the LANSS pain scale in carpal tunnel syndrome
}

This article was published in the following Dove Press journal:

Neuropsychiatric Disease and Treatment

4 January 2013

Number of times this article has been viewed

\section{Azize Esra Gürsoy \\ Mehmet Kolukısa \\ Gülsen Babacan Yıldız \\ Gülșen Kocaman \\ Arif Çelebi \\ Abdülkadir Koçer}

Department of Neurology, Bezmialem Vakıf University, Istanbul, Turkey
Correspondence: AE Gürsoy

Department of Neurology, Bezmialem

Vakıf University, Medical Faculty, Adnan

Menderes Bulvarı, 34093 Fatih, Istanbul,

Turkey

Tel +905324679404

Email aesragursoy@gmail.com
Objective: The aim of the study was to investigate the relationship between the presence of neuropathic pain assessed by the Leeds Assessment of Neuropathic Symptoms and Signs (LANSS) scale and electrophysiological findings in patients with carpal tunnel syndrome (CTS).

Methods: We studied 124 hands with idiopathic CTS with pain complaints involving hand and wrist. All hands were assessed by the LANSS with which a score of 12 or more is defined as pain dominated by neuropathic mechanisms. These hands were assigned to minimal, mild, moderate, severe, or extreme severe groups according to the results of the median nerve conduction studies. Results: A LANSS score $\geq 12$, suggestive of pain dominated by neuropathic mechanisms, was defined in 59 (47.6\%) CTS hands. Pain intensity was significantly higher in CTS hands with a LANSS score $\geq 12$ ( $P<0.001)$. Among electrophysiological findings, compound muscle action potential amplitude was significantly lower in hands with a LANSS score $\geq 12$ compared with hands with a LANSS score $<12(P=0.020)$. Severity of CTS was not significantly different between LANSS $\geq 12$ and LANSS $<12$ groups. Electrophysiological severity was significantly higher in CTS hands with evoked pain $(P=0.005)$ and allodynia $(P<0.001)$ in LANSS subscore analysis.

Conclusion: We suggest that the presence of pain dominated by neuropathic mechanisms in CTS is not related to electrophysiological CTS severity. Neuropathic pain should be assessed carefully in patients with CTS, and an appropriate treatment plan should be chosen, taking into account the clinical and electrophysiological findings together with the true pain classification.

Keywords: electrodiagnostic evaluation, carpal tunnel syndrome, LANSS, neuropathic pain

\section{Introduction}

Carpal tunnel syndrome (CTS) is the most common entrapment neuropathy and has an estimated prevalence of $3 \%-16 \% \cdot{ }^{1-4}$ CTS is caused by compression of the median nerve beneath the transverse carpal ligament, resulting in mechanical compression and/or local ischemia. ${ }^{5}$ The diagnosis of CTS involves a combination of clinical symptoms, accurate examination, and nerve conduction studies. ${ }^{6-8}$ Primary features of CTS include numbness, tingling, burning, and pain in the hand and a reduction in grip strength and function of the affected hand. ${ }^{9}$ Symptoms including numbness, tingling, and nocturnal symptoms have been considered as more specific for nerve injury, and CTS symptoms including pain, weakness, and clumsiness are symptoms that could be associated with soft tissue injury and other musculoskeletal or joint disorders. ${ }^{10}$ Pain in CTS could be dominated by neuropathic mechanisms related to peripheral nerve lesion or could be mixed pain with contributions of nociceptive mechanisms related to underlying musculoskeletal or joint disease. Identification of pain mechanisms and 
planning an appropriate treatment for pain relief is of great importance for the improvement of quality of life.

The Leeds Assessment of Neuropathic Symptoms and Signs (LANSS) scale is a clinical-based instrument for identifying patients whose pain is dominated by neuropathic mechanisms. ${ }^{11,12}$ This scale is based on analysis of sensory description and bedside examination of sensory dysfunction. ${ }^{11}$ LANSS could give information about the relative contributions of neuropathic symptoms in the diagnostic process. ${ }^{11,12}$

There are conflicting results about the correlation between severity of electrophysiological findings and symptoms in patients with CTS. ${ }^{10,13-16}$ To the best of our knowledge, no previous studies have investigated if pain dominated by neuropathic mechanisms assessed by LANSS is related to electrophysiological findings in CTS. Therefore the purpose of this study was to evaluate differences in electrophysiological findings among patients with painful CTS assessed and classified by LANSS.

\section{Materials and methods}

We recruited 120 patients who had been referred to the electrophysiology laboratory between February and May 2012 for suspected CTS and who had numbness and/or tingling in the median nerve distribution in the hands and pain in the hand and wrist with a minimum symptom duration of 3 months. Sensory deficits in the median nerve distribution, motor deficit, or hypotrophy of the median-innervated muscles and Tinel's and Phalen's signs were examined in all patients. The diagnosis of clinical CTS was made on the basis of the presence of paresthesia, pain, swelling, weakness, or clumsiness of the hand provoked or worsened by sleep and/or a sustained hand or arm position and the presence of one or more supporting criteria, including a positive Tinel sign, Phalen sign, or median nerve sensory or motor deficit. ${ }^{17}$ Patients underwent a complete neurological examination to exclude the presence of any other neurological condition. Patients were excluded if they had any of the following: clinical signs of polyneuropathy, cervical radiculopathy, or orthopedic or rheumatologic disease; a known diagnosis of hereditary neuropathy with liability to pressure palsy, diabetes mellitus or renal failure; or a known history of alcohol abuse, or drug or toxin exposure, or if they reported previous surgery or trauma to the upper limb and/or neck. All candidates gave written informed consent, and the study was approved by the local medical ethics committee.

Electrophysiological examination was performed in all patients by one examiner who used a Keypoint electromyograph (Medtronic, Skovlunde, Denmark) and who was blinded to the results of the preceding physical examination. Limb temperature was kept above $32^{\circ} \mathrm{C}$. Sensory nerve conduction studies (NCSs) were performed with an orthodromic technique that stimulated the median nerve at digit II. Sensory nerve action potentials (SNAP) were recorded by surface electrodes placed over the wrist at a distance of $13 \mathrm{~cm}$ from the active stimulating electrode. Stimulation of the sensory nerve was characterized by a duration of $100 \mu \mathrm{s}$ and an intensity of $10-30 \mathrm{~mA}$ with the filter setting at $20 \mathrm{~Hz}-2 \mathrm{kHz}$. The distal sensory latency (DSL), sensory conduction velocity (SCV) and peak-to-peak sensory nerve action potential amplitude (SNAP) were measured. Motor NCSs were performed by stimulating the median nerve at the wrist and elbow. Stimulation of the motor nerve was characterized by a duration of $100 \mu$ s and an intensity of $30-90 \mathrm{~mA}$ with a band pass filter settled at $20 \mathrm{~Hz}-10 \mathrm{kHz}$. The median nerve compound muscle action potentials (CMAP) were recorded by surface electrodes placed over the abductor pollicis brevis muscle with median nerve stimulation $6 \mathrm{~cm}$ proximal to the active recording electrode. The distal motor latency (DML) was measured at the onset of the CMAP. We calculated the motor conduction velocity $(\mathrm{MCV})$ and baseline-to-negative peak amplitude of the CMAP. A median nerve sensory conduction velocity $<48 \mathrm{~m} /$ second and a median nerve distal motor latency $>4.2$ milliseconds were considered abnormal. When the standard tests yielded normal results, median to ulnar comparison for the fourth finger was performed and the fourth finger median and ulnar SNAP peak latency difference was calculated. A difference greater than 0.4 milliseconds was considered abnormal. Ulnar nerve sensory and motor conduction studies were done to rule out ulnar nerve involvement.

After electrophysiological confirmation of CTS and selection according to exclusion criteria, 72 patients $(47.33 \pm 11.09$ years) were included in the study. The severity of electrophysiological impairment was scored with a modified neurophysiologic grading system as follows: Minimal CTS, standard negative hands with abnormal comparative tests; Mild CTS, slowing of median digit-wrist segment and normal DML; Moderate CTS, slowing of median digit-wrist segment and abnormal DML; Severe CTS, absence of median SNAPS (digit-wrist segment) and abnormal DML; Extreme severe CTS, absence of thenar motor (and sensory) response. ${ }^{18}$

After clinical and electrophysiological diagnosis of CTS, all hands with pain were assessed by the Turkish version of the LANSS pain scale. On the LANSS pain scale, a score 
of 12 or more is classified as pain dominated by neuropathic mechanisms (LANSS $\geq 12$ group), and a score of $<12$ is classified as pain not suggestive of neuropathic mechanisms (LANSS $<12$ group). The patients were asked to rate their average pain in the last month on a numeric rating scale from 0 (no pain) to 10 (extreme pain).

\section{Statistical analysis}

Data concerning clinical variables and nerve conduction studies were processed using Microsoft Office Excel and Access 2003 (Microsoft Corporation, Redmond, WA, USA), and all statistical analyses were performed using SPSS software (v 16.0; IBM Corporation, Armonk, NY, USA). Comparisons between patient groups were performed with a $t$-test , $\chi^{2}$ test or Mann-Whitney $U$ test, as appropriate. For statistical analysis of demographic characteristics, each patient with bilateral CTS was entered once. Analysis of correlation was made with Spearman's rank correlation coefficient. We carried out a linear regression analysis on the association between the LANSS score and neurophysiologic stage and pain intensity as scored by a numeric rating scale.

\section{Results}

We enrolled 72 CTS patients (six [8.33\%] men and 66 [91.67\%] women) for a total of 124 CTS hands. The mean age of patients was $47.33 \pm 11.09$ (range 22-78) years. CTS was found to be bilateral in $52(72.22 \%)$ patients and unilateral in $20(27.78 \%)$ patients; $66(53.23 \%)$ right and $58(46.77 \%)$ left hands were involved. Duration of the symptoms was $35.00 \pm 55.83$ (range 3-240) months. Thirty-nine hands $(31.5 \%)$ were categorized as minimal CTS, 19 (15.3\%) as mild CTS, $51(41.1 \%)$ as moderate CTS, ten (8.1\%) as severe CTS, and five $(4.0 \%)$ as extreme CTS. A LANSS score $\geq 12$ (suggestive of neuropathic mechanisms) was found in $59(47.6 \%)$ CTS hands, whereas a LANSS score was $<12$ in 65 (52.4\%) CTS hands. Thirty-nine patients had pain dominated by neuropathic mechanisms: ten right hands, nine left hands, and 20 bilateral. Demographic characteristics (sex, age, height, weight, and body mass index) were not significantly different between patient groups (Table 1).

Duration of symptoms was not significantly different between groups $(P=0.117)$. Sensory deficits in median nerve distribution, Tinel's sign, and Phalen's sign were found significantly more frequently in hands with a LANSS score $\geq 12$ compared with hands with a LANSS score $<12$ ( $P<0.001, P=0.002$, and $P=0.001$, respectively; Table 2$)$. Motor deficit or hypotrophy in median innervated hand muscles was not significantly different between groups
Table I Demographic characteristics of patients with CTS, according to the LANSS score

\begin{tabular}{llll}
\hline & $\begin{array}{l}\text { LANSS } \geq \mathbf{~} \mathbf{2} \\
(\mathbf{n}=\mathbf{3 9})\end{array}$ & $\begin{array}{l}\text { LANSS }<\mathbf{~} \mathbf{2} \\
(\mathbf{n}=\mathbf{3 3})\end{array}$ & $\mathbf{P}$ \\
\hline Age (years) & $46.67 \pm 12.45$ & $48.12 \pm 9.39$ & 0.574 \\
Sex (male/female) & $5 / 34$ & $1 / 32$ & $0.209^{\dagger}$ \\
Height $(\mathrm{cm})$ & $159.85 \pm 7.48$ & $158.00 \pm 4.79$ & 0.578 \\
Weight $(\mathrm{kg})$ & $79.79 \pm 15.89$ & $77.39 \pm 12.18$ & $0.48 \mathrm{I}$ \\
BMI $\left(\mathrm{kg} / \mathrm{m}^{2}\right)$ & $30.87 \pm 5.91$ & $30.83 \pm 4.47$ & 0.968 \\
\hline
\end{tabular}

Notes: For statistical analysis for demographic characteristics, each patient with bilateral CTS was entered once. Data for continuous variables are expressed as mean \pm standard deviation; 'Pearson's $\chi^{2}$ test.

Abbreviations: CTS, carpal tunnel syndrome; LANSS, Leeds Assessment of Neuropathic Symptoms and Signs scale; BMI, body mass index.

( $P=0.215$ and $P=0.784)$. Among nerve conduction studies only median nerve CMAP amplitude was significantly different in hands with a LANSS score $\geq 12$ compared with hands with a LANSS score $<12(P=0.020)$. DML and DSL were not significantly different between groups $(P=0.214, P=0.180$, respectively; Table 3$)$. Severity of electrophysiological impairment was not significantly different in the hands with a LANSS score $\geq 12$ and $<12$ $(P=0.177$; Figure 1). Pain intensity was significantly higher in hands with a LANSS score $\geq 12$ compared with hands with a LANSS score $<12(P<0.001)$. The LANSS score was significantly correlated with median nerve CMAP amplitude (Spearman's rho $=-0.286, P<0.001$ ) and pain intensity (Spearman's rho $=0.712, P<0.001$ ).

There was no correlation with LANSS score for severity of electrophysiological impairment (Spearman's rho $=-0.121$, $P=0.181)$, age $(-0.033, P=0.714)$, duration of symptoms $(0.580, P=0.520)$, DSL $(-0.150, P=0.096)$, DML $(-0.112$, $P=0.214)$, SNAP $(-0.157, P=0.082), \operatorname{SCV}(-0.088$, $P=0.329)$, and $\mathrm{MCV}(-0.062, P=0.492)$.

Linear regression analysis of the LANSS score and electrophysiological grades showed no significant association,

Table 2 Clinical findings of CTS hands with LANSS $\geq 12$ and with LANSS $<12$

\begin{tabular}{|c|c|c|c|}
\hline & $\begin{array}{l}\text { CTS hands } \\
\text { with LANSS } \\
\geq 12(n=59)\end{array}$ & $\begin{array}{l}\text { CTS hands } \\
\text { with LANSS } \\
<12(n=65)\end{array}$ & $P$ \\
\hline $\begin{array}{l}\text { Sensory deficit } \\
\text { in median nerve } \\
\text { distribution }\end{array}$ & $53(89.8 \%)$ & $38(58.46 \%)$ & $<0.001 *$ \\
\hline Motor deficit & 18 (30.5I\%) & $13(20 \%)$ & 0.215 \\
\hline Thenar atrophy & 8 (13.56\%) & 7 ( $10.77 \%)$ & 0.784 \\
\hline Tinel's sign & 38 (64.4I\%) & 24 (36.92\%) & $0.004 *$ \\
\hline Phalen's sign & 51 (86.44\%) & 38 (58.46\%) & $0.001 *$ \\
\hline
\end{tabular}

Notes: *Pearson's $\chi^{2}$ test, $P<0.05$.

Abbreviations: CTS, carpal tunnel syndrome; LANSS, Leeds Assessment of Neuropathic Symptoms and Signs scale. 
Table 3 Median nerve conduction study findings in painful CTS hands with LANSS $\geq 12$ and with LANSS $<12$

\begin{tabular}{lccc}
\hline & $\begin{array}{l}\text { CTS hands } \\
\text { with LANSS } \\
\geq \mathbf{I 2}(\mathbf{n}=\mathbf{5 9})\end{array}$ & $\begin{array}{l}\text { CTS hands } \\
\text { with LANSS } \\
<\mathbf{I 2}(\mathbf{n}=\mathbf{6 5})\end{array}$ & \\
\hline DSL (millisecond) & $3.52 \pm 0.67$ & $3.74 \pm 0.93$ & 0.18 \\
SNAP amplitude $(\mu \mathrm{V})$ & $12.75 \pm 9.53$ & $14.52 \pm 8.10$ & 0.26 \\
SCV (m/second) & $44.74 \pm 8.89$ & $43.16 \pm 9.56$ & 0.38 \\
DML (millisecond) & $4.53 \pm 1.66$ & $4.21 \pm 1.09$ & 0.21 \\
CMAP amplitude (mV) & $6.09 \pm 2.47$ & $7.14 \pm 2.47$ & $0.02^{*}$ \\
MCV(m/second) & $55.30 \pm 5.35$ & $55.35 \pm 5.10$ & 0.96 \\
\hline
\end{tabular}

Note: $* P<0.05$.

Abbreviations: CTS, carpal tunnel syndrome; LANSS, Leeds Assessment of Neuropathic Symptoms and Signs scale; DSL, distal sensory latency; SNAP, sensory nerve action potential; SCV, sensory nerve conduction velocity; DML, distal motor latency; CMAP, compound muscle action potential; MCV, motor nerve conduction velocity.

whereas LANSS score and pain intensity showed a significant linear association between the two (B [1.369], $t=10.663$; $P<0.001)$.

Painful CTS hands with a LANSS score $\geq 12$ suggestive of neuropathic mechanisms had significantly more frequent autonomic symptoms (LANSS Item 2; $P<0.001$ ), evoked pain (LANSS Item 3; $P<0.001$ ), paroxysmal pain (LANSS Item $4 ; P<0.001$ ), thermal pain (LANSS Item 5; $P<0.001$ ), allodynia (LANSS Item $6 ; P=0.002$ ), and altered pinprick threshold (LANSS Item 7; $P<0.001$ ) compared with hands with a LANSS score $<12$ (Table 4).

The electrophysiological stage of CTS hands with evoked pain (LANSS Item 3) and with allodynia (LANSS Item 6) was significantly higher compared with hands without evoked pain and allodynia ( $P=0.005$ and $P<0.001$, respectively) as shown in Table 5. In CTS hands with evoked pain, DSL was significantly longer (3.49 \pm 1.10 milliseconds vs $2.7 \pm 1.74$ milliseconds, $P=0.002)$, SNAP was significantly lower $(10.43 \pm 9.26 \mu \mathrm{V}$ vs $15.59 \pm 7.85 \mu \mathrm{V}, P<0.001)$,

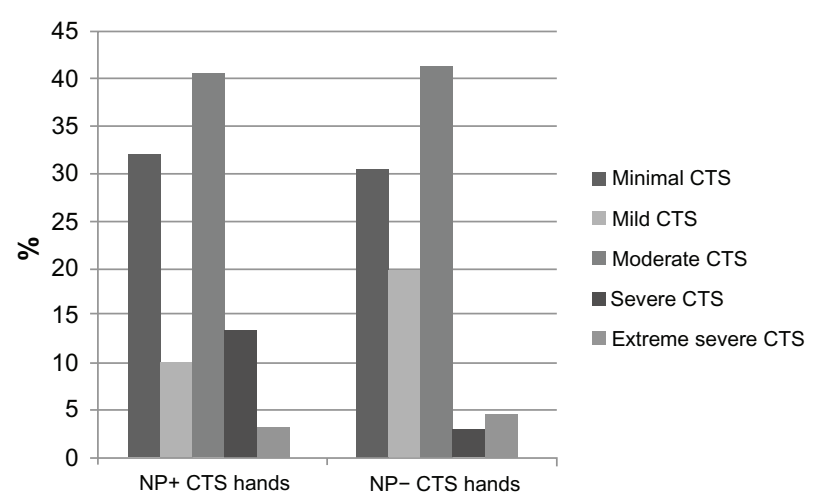

Figure I Percentage of CTS in different severity stages in NP+ and NP- CTS hands. Abbreviations: CTS, carpal tunnel syndrome; NP+, neuropathic pain positive according to Leeds Assessment of Neuropathic Symptoms and Signs (LANSS) scale; NP-, neuropathic pain negative according to LANSS.
Table 4 Frequency of the LANSS items in CTS hands with LANSS $\geq 12$ and LANSS $<12$

\begin{tabular}{lll}
\hline & $\begin{array}{l}\text { LANSS } \geq \mathbf{I 2} \\
(\mathbf{n}=\mathbf{5 9})\end{array}$ & $\begin{array}{l}\text { LANSS }<\text { I2 } \\
\mathbf{( n = 6 5 )}\end{array}$ \\
\hline Dysesthesia (Item I) & $59(100 \%)$ & $65(100 \%)$ \\
Autonomic dysfunction (Item 2) & $25(42.4 \%)$ & $0(0 \%)$ \\
Evoked pain (Item 3) & $39(66.1 \%)$ & $7(10.8 \%)$ \\
Paroxysmal (Item 4) & $30(50.8 \%)$ & $8(12.3 \%)$ \\
Thermal (Item 5) & $44(74.6 \%)$ & $19(29.2 \%)$ \\
Allodynia (Item 6) & $8(13.6 \%)$ & $0(0 \%)$ \\
Altered PPT (Item 7) & $59(100 \%)$ & $49(72.3 \%)$ \\
\hline
\end{tabular}

Abbreviations: CTS, carpal tunnel syndrome; LANSS, Leeds Assessment of Neuropathic Symptoms and Signs scale; PPT, pinprick threshold.

SCV was significantly slower $(31.60 \pm 10.59 \mathrm{~m} /$ second vs $42.67 \pm 4.49 \mathrm{~m} /$ second, $P<0.001)$, and CMAP was significantly lower $(5.71 \pm 2.54 \mathrm{mV}$ vs $7.2 \pm 2.32 \mathrm{mV}, P<0.001)$ compared with CTS hands without evoked pain.

In CTS hands with allodynia, DSL was significantly longer $(3.86 \pm 1.08$ milliseconds vs $2.94 \pm 0.74$ milliseconds, $P=0.003)$, SNAP was significantly lower $(5.36 \pm 7.18 \mu \mathrm{V}$ vs $14.25 \pm 8.56 \mu \mathrm{V}, P=0.005)$, SCV was significantly slower $(21.59 \pm 23.42 \mathrm{~m} /$ second vs $39.74 \pm 15.69 \mathrm{~m} /$ second, $P=0.003)$, and CMAP was significantly lower $(4.74 \pm 2.49 \mathrm{mV}$ vs $6.78 \pm 2.46 \mathrm{mV}, P=0.025)$ compared with CTS hands without allodynia. In CTS hands with altered pinprick threshold, only CMAP was significantly lower $(6.43 \pm 2.53 \mathrm{mV}$ vs $7.96 \pm 1.92 \mathrm{mV}, P=0.016$ ) compared with CTS hands without altered PPT. We found no significant difference for electrophysiological findings in other LANSS subscore analyses.

\section{Discussion}

Most patients with CTS seek medical attention because of pain. Early identification, true classification, and understanding of underlying mechanisms of pain are necessary for an appropriate treatment plan. ${ }^{19}$ The Turkish version of the LANSS pain scale is a validated tool for distinguishing patients with neuropathic pain from those with nociceptive pain. ${ }^{12}$ The LANSS pain scale developed by Bennett ${ }^{11}$ is a seven-item scale consisting of five questions about sensory experiences, including dysesthesia, autonomic dysfunction, evoked pain, paroxysmal pain, and thermal pain and two items based on a bedside examination (allodynia and pinprick threshold) of the painful area. Although Backonja et al suggested that the LANSS pain scale might not be appropriate for those patients with symmetrical neuropathic presentations, we used it in patients with bilateral painful CTS and used an adjacent non-painful area as the control, as described by Bennett. ${ }^{11,20}$ Our results showed that neuropathic pain based on LANSS may be found in $47.6 \%$ of CTS hands. 
Table 5 Comparison of electrophysiological stages and responses to each LANSS item

\begin{tabular}{|c|c|c|c|}
\hline & $\begin{array}{l}\text { Electrophysiological stage (median } \pm \text { SD) } \\
\text { with positive response }\end{array}$ & $\begin{array}{l}\text { Electrophysiological stage (median } \pm \mathrm{SD} \text { ) } \\
\text { with negative response }\end{array}$ & $P$ \\
\hline Dysesthesia (Item I) & $2 \pm 1.990(n=124)$ & & \\
\hline Autonomic dysfunction (Item 2) & $2 \pm 1.098(n=25)$ & $3 \pm 1.090(n=99)$ & 0.078 \\
\hline Evoked pain (Item 3) & $3 \pm 1.219(n=46)$ & $2 \pm 0.963(n=78)$ & $0.005^{*}$ \\
\hline Paroxysmal (Item 4) & $2.5 \pm 1.119(n=38)$ & $3 \pm 1.091(n=86)$ & 0.3 \\
\hline Thermal (Item 5) & $3 \pm 1.184(n=63)$ & $3 \pm 1.009(n=61)$ & 0.417 \\
\hline Allodynia (Item 6) & $3.5 \pm 0.535(n=8)$ & $2.5 \pm 1.086(n=116)$ & $0.001 *$ \\
\hline Altered PPT (Item 7) & $3 \pm 1.119(n=106)$ & $2 \pm 0.963(n=18)$ & 0.292 \\
\hline
\end{tabular}

Notes: $P$-value from Mann-Whitney test; $* P<0.05$.

Abbreviations: LANSS, Leeds Assessment of Neuropathic Symptoms and Signs scale; SD, standard deviation; PPT, pinprick threshold.

A feature of our study was the analysis of the various clinical qualities of neuropathic pain in CTS evaluated by LANSS. We showed that autonomic dysfunction, evoked pain, paroxysmal pain, thermal pain, and allodynia are less frequent than dysesthesia and hypoesthesia on pinprick examination in painful CTS hands. As expected, all of our patients had a positive response to LANSS Item 1 (dysesthesia) caused by abnormal non-nociceptive $A \beta$-fiber activity.

There are conflicting results about the relationship of pain with clinical and electrophysiological findings. Similar to our results, Truini et al found no difference in the frequency of the different CTS severity grades between neuropathic and non-neuropathic groups. ${ }^{21}$ Clinical pain parameters and Boston Carpal Tunnel Syndrome Questionnaire (BCTQ) scores were not significantly different among patients with minimal, moderate, and severe CTS. ${ }^{22}$ However, there was a statistically significant correlation between the total Washington neuropathic pain scale score and severity of CTS. ${ }^{23}$ OrtizCorredor et al reported no significant correlation between pain status evaluated with BCTQ and nerve conduction studies in CTS patients. ${ }^{24}$ Although we did not find a significant difference in the frequency of motor deficit or hypotrophy in median innervated hand muscles by clinical evaluation between groups, we found significantly reduced median nerve CMAP amplitudes in CTS hands with a LANSS score $\geq 12$ compared to a LANSS score $<12$. One reason for the discrepancy between the clinical and electrophysiological results in our study could be that the mean CMAP amplitudes in CTS hands with a LANSS score $\geq 12$ were not actually below normal limits, although they were significantly lower compared to the LANSS $<12$ group. In a previous study, nocturnal pain frequency was found to be related to decreased median CMAP amplitudes, ${ }^{25}$ and hand weakness and clumsiness have been reported to be related to the severity of sensory symptoms, including pain, numbness, and tingling, while hand clumsiness has been found to be related to median nerve motor involvement. ${ }^{26}$
We showed in the LANSS subscore analysis that CTS hands with evoked pain and allodynia have more severe electrophysiological impairment concordant with more severe $A \beta$ fiber dysfunction. Ongoing pain intensity has been reported to be significantly correlated to the dysfunction of thick myelinated motor nerve fibers, ie, prolonged DML and reduced CMAP. ${ }^{27}$ However, Truini et al reported that provoked pain did not correlate with neurophysiological data. ${ }^{21}$ Further studies should be done to investigate the contribution of thick myelinated fiber dysfunction to pain mechanisms in CTS.

There are conflicting results about the involvement of $A \delta$ and C fibers in neuropathic pain in CTS. ${ }^{28,29}$ De Tommaso et al found an increased pain threshold after laser stimulation of the second and third fingers, with normal pain thresholds at the fifth finger and hand dorsum levels in patients presenting with CTS symptoms and they suggested that their findings confirm the peripheral involvement of the median nerve nociceptive afferents. ${ }^{29}$ In a recent study, no significant differences were found in pain parameters, including pressure pain thresholds, warm and cold detection thresholds, and heat and cold pain thresholds, among patients with minimal, moderate, and severe CTS. ${ }^{22}$ Extramedian spread of sensory symptoms, hyperalgesia enlarged outside the median nerve zone, bilateral heightened pain sensitivity in unilateral CTS, and altered cortical hand somatotophy associated with CTS suggest that central nervous system alterations in pain processing accompany peripheral dysfunction..$^{30-36}$

The results presented herein demonstrate that the presence of evoked pain and allodynia might be associated with more severe electrophysiological impairment, although neuropathic pain assessed with a total LANSS score is not correlated with electrophysiological CTS severity. Therefore, neuropathic pain in patients with CTS should be handled and treated independently from electrophysiological data. 


\section{Disclosure}

The authors report no conflicts of interest in this work.

\section{References}

1. Stevens JC. The electrodiagnosis of carpal tunnel syndrome. Muscle Nerve. 1997;20:1477-1486.

2. de Krom MC, Knipschild PG, Kester AD, Thijs CT, Boekkooi PF, Spaans F. Carpal tunnel syndrome: prevalence in the general population. J Clin Epidem. 1992;45:373-376.

3. Atroshi I, Gummesson C, Johnsson R, Ornstein E, Ranstam J, Rosen I. Prevalence of carpal tunnel syndrome in a general population. JAMA. 1999;282:153-158.

4. Aroori S, Spence R. Carpal tunnel syndrome. Ulster Med J. 2008;77: 6-17.

5. Werner RA, Andary M. AANEM Monograph: electrodiagnostic evaluation of carpal tunnel syndrome. Muscle Nerve. 2011;44: 597-607.

6. Rempel D, Evanoff B, Amadio PC, et al. Consensus criteria for the classification of carpal tunnel syndrome in epidemiologic studies. Am J Public Health. 1998;88(10):1447-1451.

7. Jablecki CK, Andary MT, Floeter MK, et al; American Association of Electrodiagnostic Medicine; American Academy of Neurology; American Academy of Physical Medicine and Rehabilitation. Practice parameter: electrodiagnostic studies in carpal tunnel syndrome. Report of the American Association of Electrodiagnostic Medicine, American Academy of Neurology, and the American Academy of Physical Medicine and Rehabilitation. Neurology. 2002;58(11):1589-1592.

8. Keith MW, Masear V, Chung K, et al. Diagnosis of carpal tunnel syndrome. J Am Acad Orthop Surg. 2009;17(6):389-396.

9. Padua L, Padua R, Lo Monaco M, Aprile I, Tonali P. Multiperspective assessment of carpal tunnel syndrome: a multicenter study. Italian CTS Study Group. Neurology. 1999;53:1654-1659.

10. You H, Simmons Z, Freivalds A, Kothari MJ, Naidu SH. Relationships between clinical symptom severity scales and nerve conduction measures in carpal tunnel syndrome. Muscle Nerve. 1999;22:497-501.

11. Bennett M. The LANSS Pain Scale: the Leeds Assessment of Neuropathic Symptoms And Signs. Pain. 2001;92:147-157.

12. Yücel A, Şenocak M, Kocasoy Orhan E, Çimen A, Ertaş M. Results of the Leeds Assessment of Neuropathic Symptoms and Signs Pain Scale in Turkey: a validation study. J Pain. 2004;8:427-432.

13. Dhong ES, Han SK, Lee BI, Kim WK. Correlation of electrodiagnostic findings with subjective symptoms in carpal tunnel syndrome. Ann Plast Surg. 2000;45:127-131.

14. Longstaff L, Milner RH, O'Sullivan S, Fawcett P. Carpal tunnel syndrome: the correlation between outcome, symptoms and nerve conduction study findings. J Hand Surg Br. 2001;26:475-480.

15. Schrijver HM, Gerritsen AA, Strijers RL, et al. Correlating nerve conduction studies and clinical outcome measures on carpal tunnel syndrome: lessons from a randomized controlled trial. J Clin Neurophysiol. 2005;22:216-221.

16. Chan L, Turner JA, Comstock BA, et al. The relationship between electrodiagnostic findings and patient symptoms and function in carpal tunnel syndrome. Arch Phys Med Rehabil. 2007;88:19-24.

17. [No authors listed]. Practice parameter for carpal tunnel syndrome (summary statement). Report of the Quality Standards Subcommittee of the American Academy of Neurology. Neurology. 1993;43: 2406-2409.

18. Padua L, Lomonaco M, Gregori B, Valente EM, Padua R, Tonali P. Neurophysiological classification and sensitivity in 500 carpal tunnel syndrome hands. Acta Neurol Scand. 1997;96:211-217.
19. Bennett GJ. Chronic pain due to peripheral nerve damage: an overview. In: Fields HL, Liebeskind JC, editors. Progress in Pain Research and Management. Seattle: IASP Press; 1994a;1:51-59.

20. Backonja MM. Need for differential assessment tools of neuropathic pain and the deficits of LANSS pain scale. Pain. 2002;98:229-230.

21. Truini A, Padua L, Biasiotta A, et al. Differential involvement of A-delta and A-beta fibres in neuropathic pain related to carpal tunnel syndrome. Pain. 2009;145(1-2):105-109.

22. de la Llave-Rincón AI, Fernández-de-las-Peñas C, Laguarta-Val S, et al. Increased pain sensitivity is not associated with electrodiagnostic findings in women with carpal tunnel syndrome. Clin J Pain. 2011; 27(9):747-754.

23. Oncel C, Bir LS, Sanal E. The relationship between electrodiagnostic severity and Washington Neuropathic Pain Scale in patients with carpal tunnel syndrome. Agri. 2009;21(4):146-148.

24. Ortiz-Corredor F, Calambas N, Mendoza-Pulido C, Galeano J, Díaz-Ruíz J, Delgado O. Factor analysis of carpal tunnel syndrome questionnaire in relation to nerve conduction studies. Clin Neurophysiol. 2011;122(10):2067-2070.

25. Koçer A, Türk Börü Ü. Pain frequency at night reflects median nerve injury in carpal tunnel syndrome. Turk $J$ Phys Med Rehab. 2005;51(4):138-144.

26. Tamburin S, Cacciatori C, Marani S, Zanette G. Pain and motor function in carpal tunnel syndrome: a clinical, neurophysiological and psychophysical study. J Neurol. 2008;255(11):1636-1643.

27. Lang E, Claus D, Neundörfer B, Handwerker HC. Parameters of thick and thin nexweofiber functions as predictors of pain in carpal tunnel syndrome. Pain. 1995;60:295-302.

28. Dahlin LB, Shyu BC, Danıelsen N, Andersson SA. Effects of nerve compression or ischaemia on conduction proprieties of myelinated and non-myelinated nerve fibres. An experimental study in the rabbit common peroneal nerve. Acta Physiol Scand. 1989;136:97-105.

29. de Tommaso M, Libro G, Difruscolo O, et al. Laser evoked potentials in carpal tunnel syndrome. Clin Neurophysiol. 2009;120(2):353-359.

30. Tecchio F, Padua L, Aprile I, Rossini PM. Carpal tunnel syndrome modifies sensory hand cortical somatotopy: a MEG study. Hum Brain Mapp. 2002;17(1):28-36.

31. de la Llave-Rincón AI, Fernández-de-las-Peñas C, Fernández-Carnero J, Padua L, Arendt-Nielsen L, Pareja JA. Bilateral hand/wrist heat and cold hyperalgesia, but not hypoesthesia, in unilateral carpal tunnel syndrome. Exp Brain Res. 2009;198(4):455-463.

32. Fernández-de-las-Peñas C, de la Llave-Rincón AI, Fernández-Carnero J, Cuadrado Ll, Arendt-Nielsen L, Pareja JA. Bilateral widespread mechanical pain sensitivity in carpal tunnel syndrome: evidence of central processing in unilateral neuropathy. Brain. 2009;132(Pt 6):1472-1479.

33. Tamburin S, Cacciatori C, Praitano ML, Marani S, Zanette G. Ulnar nerve impairment at the wrist does not contribute to extramedian sensory symptoms in carpal tunnel syndrome. Clin Neurophysiol. 2009;120(9): $1687-1692$.

34. Fernández-de-las-Peñas C, Madeleine P, Martínez-Perez A, ArendtNielsen L, Jiménez-García R, Pareja JA. Pressure pain sensitivity topographical maps reveal bilateral hyperalgesia of the hands in patients with unilateral carpal tunnel syndrome. Arthritis Care Res (Hoboken). 2010;62(8):1055-1064.

35. Zanette G, Cacciatori C, Tamburin S. Central sensitization in carpal tunnel syndrome with extraterritorial spread of sensory symptoms. Pain. 2010;148(2):227-236.

36. Bialosky JE, Bishop MD, Robinson ME, Price DD, George SZ. Heightened pain sensitivity in individuals with signs and symptoms of carpal tunnel syndrome and the relationship to clinical outcomes following a manual therapy intervention. Man Ther. 2011;16(6):602-608. 
Neuropsychiatric Disease and Treatment

Dovepress

\section{Publish your work in this journal}

Neuropsychiatric Disease and Treatment is an international, peerreviewed journal of clinical therapeutics and pharmacology focusing on concise rapid reporting of clinical or pre-clinical studies on a range of neuropsychiatric and neurological disorders. This journa is indexed on PubMed Central, the 'PsycINFO' database and CAS.

The manuscript management system is completely online and includes a very quick and fair peer-review system, which is all easy to use. Visit http://www.dovepress.com/testimonials.php to read real quotes from published authors.

Submit your manuscript here: http://www.dovepress.com/neuropsychiatric-disease-and-treatment-journal 